\title{
El Consentimiento con el Otro en la Interpretación de la Comprensión en Matemáticas
}

\author{
Consent with the Other in the Interpretation of Mathematical \\ Understanding
}

\author{
Jesús Gallardo Romero* \\ Verónica A. Quintanilla Batallanos ${ }^{* *}$
}

\begin{abstract}
Resumen
Reconocer la actividad matemática escolar como un proceso interpretativo nos obliga a dar una respuesta operativa al problema de la referencia en la interpretación. En este trabajo proponemos situarla en el consentimiento con el otro, un rastro visible complementario al uso del conocimiento matemático que incorporamos a la dimensión hermenéutica de un modelo en desarrollo para la interpretación de la comprensión en matemáticas. El consentimiento con el otro nos permite, además, completar de un modo operativo el ciclo interpretativo de la comprensión de la actividad matemática en el aula. En la práctica, ponemos de manifiesto su presencia con evidencias de los acuerdos que alcanzan dos alumnas de primer curso de educación secundaria con su profesor y sus compañeras durante la resolución de una tarea de divisibilidad de números naturales. Finalizamos la exposición, justificando de qué manera el consentimiento con el otro puede vincularse en nuestro modelo interpretativo con la dimensión social y afectiva de la comprensión en matemáticas.
\end{abstract}

Palabras-clave: Afecto en Matemáticas. Aproximación Discursiva. Aritmética Escolar. Divisibilidad de Números Naturales. Educación Matemática.

\begin{abstract}
Recognizing the mathematical activity as an interpretative process forces us to give an operative answer to the problem of reference in the interpretation. In this work, we propose to situate this reference in the consent with the other, a visible trace complementary to the use of the mathematical knowledge that we include in the hermeneutical dimension of an operative model for interpreting understanding in mathematics. The consent with the other enables us to complete the interpretative cycle of understanding of mathematical activity in the classroom. In practice, this consent is shown through agreements between two secondary students with their teacher and their peers in solving a task of divisibility of natural numbers. We conclude justifying how the consent with the other can be linked from our interpretative model to the social and affective dimension of understanding in mathematics.
\end{abstract}

Keywords: Affect in Mathematics. Discursive Approach. Divisibility of Natural Numbers. Mathematics Education. School Arithmetics.

\footnotetext{
* Doctor en Didáctica de la Matemática por la Universidad de Málaga (UMA). Profesor de Didáctica de la Matemática. Departamento de Didáctica de la Matemática, de las Ciencias Sociales y de las Ciencias Experimentales de la Universidad de Málaga, Málaga, España. Dirección postal: Facultad de Ciencias de la Educación, Campus de Teatinos, 29071, Málaga, España.E-mail: gallardoromero@uma.es.

** Máster en Políticas y Prácticas de Innovación Educativa por la Universidad de Málaga (UMA). Profesora de Didáctica de la Matemática. Departamento de Didáctica de la Matemática, de las Ciencias Sociales y de las Ciencias Experimentales de la Universidad de Málaga, Málaga, España. Dirección postal: Facultad de Ciencias de la Educación, Campus de Teatinos, 29071, Málaga, España. E-mail: veronicaquintanilla@uma.es.
} 


\section{Introducción}

Los procesos involucrados en la enseñanza y el aprendizaje de las matemáticas transcurren en entornos esencialmente interpretativos, donde los alumnos y el profesor son intérpretes de una actividad matemática, propia y ajena, mediada por un contexto social y cultural (BROWN, 1996; ELL, 2006; SÁENZ-LUDLOW; ZELLWEGER, 2012). En el trabajo cotidiano, en el aula de matemáticas, abundan los escenarios y los momentos donde el estudiante interpreta los conocimientos matemáticos y las situaciones en los que éstos se ponen en juego. Los estudiantes se interpretan entre sí en procesos discursivos y de comunicación entre iguales e, igualmente, interpretan la actividad docente desplegada por el profesor. Éste, por su parte, interpreta la actividad matemática que realizan los estudiantes, así como los propios conocimientos matemáticos que enseña a través de sus propuestas didácticas (RESÉNDIZ, 2010). Y, si además lo que se persigue es el estudio de los procesos interpretativos desplegados en tales escenarios, hay que contar, también, con la presencia del correspondiente agente externo encargado de llevar a cabo la interpretación.

En los últimos años nos hemos interesado por el estudio de uno de estos procesos interpretativos. El de la interpretación por parte de un agente externo (profesor o investigador) de la comprensión matemática de los estudiantes a partir de la actividad que manifiestan cuando se enfrentan a situaciones problemáticas que requieren el uso del conocimiento matemático objeto de comprensión (GALLARDO; GONZÁLEZ, 2006; GALLARDO; GONZÁLEZ; QUISPE, 2008; GALLARDO; GONZÁLEZ; QUINTANILLA, 2013, 2014a). Toda observación sobre el quehacer matemático de los alumnos, realizada con el fin de extraer información sobre su comprensión, ha de ser interpretada por quien efectúa la observación (MORGAN; WATSON, 2002).

De este modo, se van configurando en el aula distintos escenarios de valoración de la comprensión, cada uno (una vez más) con particularidades específicas, donde la interpretación de la actividad matemática observable que el estudiante despliega en ellos desempeña un papel esencial. La cuestión fundamental a la que nos enfrentamos en el contexto de la actividad escolar como proceso interpretativo es: ¿cómo podemos interpretar la comprensión de los estudiantes a partir de su actividad matemática observable? Esta cuestión básica y genérica se materializa en distintas problemáticas, específicas y relacionadas, que son las que delimitan y dirigen la presente investigación.

La primera de ellas atañe al problema de la objetividad de la interpretación y de la consiguiente referencia a considerar para llevarla a cabo. En el apartado 2 de este trabajo 
afrontamos esta cuestión, relacionando las principales orientaciones sobre la interpretación de la comprensión en matemáticas y presentando una propuesta integradora que tiene como referencia interpretativa inicial los usos dados a los conocimientos matemáticos ante situaciones problemáticas pertenecientes a su ámbito fenómeno-epistemológico. Con objeto de solventar de forma operativa el problema derivado que supone el retorno a la propia comprensión matemática del estudiante, también proponemos, en la parte central del trabajo, la búsqueda del consentimiento con el otro como referencia complementaria a la de los usos del conocimiento matemático.

Así, en el apartado 3 exponemos la justificación y configuración de la nueva fase de consentimiento introducida en el proceso de interpretación de la comprensión matemática según nuestro modelo operativo. Y, en el apartado 4, hacemos patente la presencia del consentimiento en la práctica a través de los ejemplos concretos de dos alumnas de primero de educación secundaria, en los que se aprecian los detalles de la aplicación del ciclo interpretativo completo. Finalmente, en el apartado 5 argumentamos en favor de una vía de acceso al desarrollo de la faceta socioafectiva de la actividad matemática en el aula, mediante la búsqueda del consentimiento con el otro, que siempre queda mediatizada por la propia comprensión matemática del estudiante.

\section{Objetividad y referencia en la interpretación de la comprensión en matemáticas}

La interpretación de la actividad humana requiere de una intervención intencional por parte del intérprete que siempre se ve afectada por el problema abierto de la objetividad. ¿Es posible participar en un proceso de comunicación y entendimiento sin influir, o influyendo lo mínimo posible, sobre el propio proceso que se pretende comprender? En el ámbito de las ciencias sociales, el conocimiento generado por cualquier propuesta interpretativa siempre estará condicionado por la teoría con la que se interpreta (POPPER, 2005). El intérprete participa e influye en el procedimiento de interpretación, y cualquier anticipación del sentido forma parte integrante del propio sentido a interpretar (HABERMAS, 1999; RICEUR, 2002). Esta circunstancia no hay que entenderla, en todo caso, como una debilidad, sino que forma parte de la propia naturaleza de la interpretación. Más que una limitación ineludible en el estudio de la comprensión, la interpretación se erige como la vía operativa necesaria para el acceso efectivo a la misma. El reto consiste en buscar referencias con las que poder garantizar un cierto grado de plausibilidad en los conocimientos adquiridos. La pertinencia de las 
propuestas interpretativas habría que buscarla no tanto en alguna supuesta veracidad adquirida, sino en términos de su aplicabilidad o potencialidad práctica (TAHTA, 1996).

¿Dónde situar la referencia para la interpretación de la comprensión en matemáticas? En Educación Matemática encontramos diferentes orientaciones que dan respuestas distintas a esta cuestión, en función del lugar hacia donde apunte la interpretación: al alumno que se pronuncia, a la actividad registrada elaborada por él o a alguna otra referencia externa más allá de todo registro escrito (Figura 1).

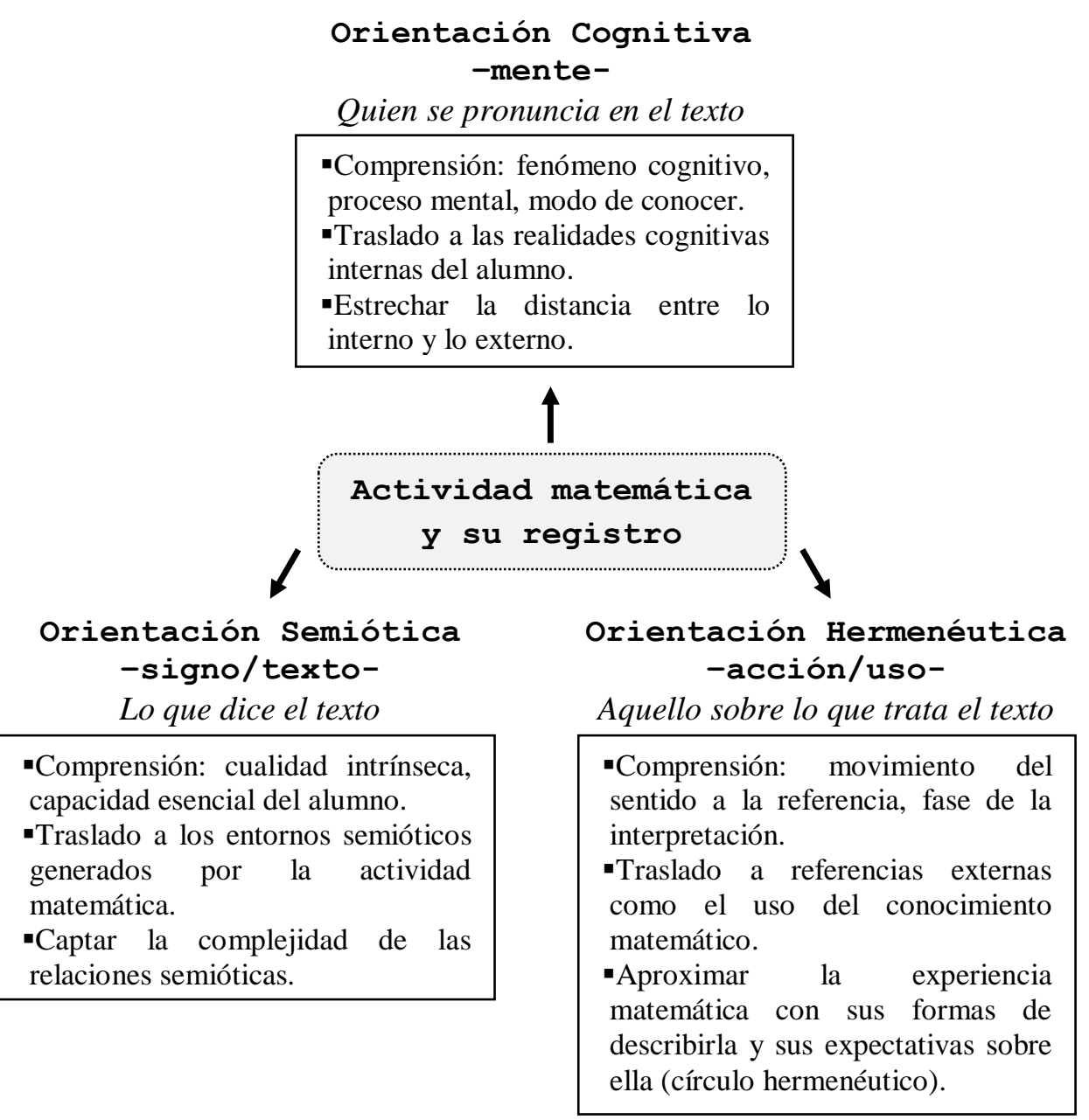

Figura 1 - Orientaciones en la interpretación de la comprensión en matemáticas

En la orientación cognitiva, la interpretación se presenta como un traslado hacia la esfera mental del estudiante, a la que pertenece su comprensión matemática, tomando como vía las distintas manifestaciones observables generadas durante su quehacer matemático. En esta orientación, interpretar supone acceder a realidades cognitivas internas con ayuda de la observación de realizaciones sensibles objetivadas. La objetividad de la interpretación se sustenta en la independencia atribuida a las producciones externas establecidas y conservadas en registros y representaciones de varios tipos, verbales y escritos. Ejemplos de esta 
orientación los encontramos en el entorno del enfoque representacional de la comprensión (BARMBY et al., 2007; GOLDIN, 2002)

La orientación semiótica asume un claro distanciamiento con el carácter mental de la comprensión. La interpretación se circunscribe al espacio exclusivo de la actividad matemática visible y del uso que en ella se hace de los sistemas de signos matemáticos. Interpretar supone trasladarse a los entornos semióticos generados por estas prácticas y producciones matemáticas observables, suspendiendo incluso cualquier referencia a la realidad externa que circunda a los propios productos semióticos. Tiene como objeto captar la complejidad de las relaciones semióticas desplegadas en las diversas acciones matemáticas observadas y registradas en los alumnos. Ejemplos de esta orientación podemos encontrarlos en el análisis semiótico incluido en el enfoque ontosemiótico de la cognición e instrucción matemática (GODINO, 2002; GODINO; BATANERO; FONT, 2007) y en la visión peirceana de la interpretación como doble proceso semiótico sugerida por Sáenz-Ludlow y Zellweger (2012).

En la orientación hermenéutica la interpretación adopta un papel más central en la comprensión matemática. El registro observable generado durante la actividad matemática y su textualización (en forma de respuestas matemáticas escritas, diálogos transcritos, acciones videograbadas) es la principal fuente depositaria de la expresión visible de la comprensión. Sin embargo, aunque la comprensión y su interpretación se sustenten en el texto, esta orientación trasciende al análisis literal y simbólico de lo puramente semiótico. La referencia última de la comprensión del estudiante no se sitúa en el registro escrito (signo o texto) sino en referencias externas como el uso del conocimiento matemático por parte del estudiante en un contexto particular. Un ejemplo de esta orientación lo encontramos en la posición postestructuralista de Brown (1996, 2001, 2008) sobre el aprendizaje matemático y el significado de la actividad en el aula.

Al afrontar la interpretación de la comprensión en matemáticas no hay que asumir como inevitable la elección entre orientaciones. Apostar por la integración frente a la selección de alternativas es otra opción en la búsqueda de interpretaciones cada vez más apropiadas. De hecho, se pueden establecer vínculos dialécticos entre las orientaciones con los que superar, o al menos reducir, las limitaciones propias de cada una, así como sus diferencias relativas (GALLARDO; GONZÁLEZ; QUINTANILLA, 2014b).

Encontramos un ejemplo de contribución integradora en el análisis cognitivo de la actividad matemática propuesto por Duval (2006), donde se justifica la necesidad de considerar representaciones semióticas en el interior de las estructuras mentales de los 
individuos (conexión cognitivo-semiótica). También consideramos integrador nuestro modelo operativo para la interpretación de la comprensión en matemáticas. En él introducimos una visión extendida de la interpretación, inspirada en parte en la dialéctica promovida por la teoría hermenéutica del texto de Ricœur (2002), donde las tres orientaciones intervienen en fases diferentes del mismo proceso interpretativo (conexión cognitivo-semióticohermenéutica).

Hasta ahora, nuestra referencia para la interpretación de la comprensión la hemos situado en el propio conocimiento matemático y en sus usos dados durante la actividad matemática. Aunque la comprensión y la interpretación se ejerzan sobre la mediación de un texto, consideramos que rebasa el campo de lo meramente semiótico. El hecho de que la capacidad para utilizar el conocimiento matemático dependa, en buena medida, de su comprensión, nos lleva a situar la referencia de la comprensión del estudiante, no solo en el registro escrito, sino en el uso del conocimiento matemático que deja entrever.

$\mathrm{Al}$ actuar, el estudiante interviene en el mundo de las prácticas matemáticas y de los fenómenos organizados por los conocimientos matemáticos puestos en uso. Esta intervención sobre la realidad matemática, sobre el mundo epistemológico y fenomenológico de los objetos matemáticos, trasciende la esfera semiótica y hace que los usos del conocimiento matemático se erijan como primera referencia extralingüística de la acción o actividad matemática. Por eso la interpretación en nuestro modelo se dirige, en un primer momento, a la exteriorización y caracterización de los usos del conocimiento matemático que se desprenden de los rastros de comprensión emergentes de los registros escritos (GALLARDO; GONZÁLEZ; QUINTANILLA, 2013, 2014a).

\section{El retorno a la comprensión matemática a través del consentimiento con el otro}

Consideramos que la comprensión en matemáticas comparte el carácter interno propio de las actividades intelectuales cognitivas específicas. Esta realidad impide, por ahora, considerar el acceso y la observación directa como opción metodológica para obtener información sobre la comprensión matemática de los estudiantes. De hecho, el estudio de la comprensión en matemáticas se ve afectado por el conocido problema de las otras mentes presente en la epistemología contemporánea (DANCY, 1993), pudiéndose ver como una concreción de éste en el ámbito particular de la Educación Matemática. En dicho problema se discute la posibilidad de aceptar algún tipo de vínculo entre el estado mental de un sujeto 
ajeno y el de su agente intérprete, así como de acceder a proposiciones sobre mentes distintas a la nuestra propia.

En cualquier caso, la imposibilidad de observar directamente la naturaleza y el funcionamiento interno de la comprensión, al tiempo que justifica la razón de ser de la interpretación, también provoca un traslado al ámbito externo de la actividad matemática observable. El centro de atención se desplaza desde el estudiante cuya comprensión se quiere valorar hacia su propia producción matemática externa. Una inevitable transición desde lo interno a lo externo que también trae consigo, como consecuencia, un distanciamiento con el propio alumno. Esto hace que las diferentes propuestas interpretativas se vean obligadas a responder a la cuestión del retorno a la comprensión matemática.

La conexión última entre los rastros visibles del conocimiento, las producciones externas y los signos objetivados empleados por los estudiantes durante el desarrollo de sus prácticas matemáticas, por un lado, y la propia comprensión matemática como actividad intelectual específica de carácter interno, por otro, todavía requiere una última concreción. Se busca recomponer la comprensión matemática que fue provisionalmente distanciada con anterioridad en la fase interpretativa correspondiente al paso de lo cognitivo a lo semiótico. En orientaciones como la cognitiva este retorno es directo pero, precisamente por ello, la interpretación se ve afectada por dificultades operativas relacionadas con el acceso a las características mentales de la comprensión, así como con el problema ontológico asociado a las representaciones internas (FONT; GODINO; D’AMORE, 2007).

Por su parte, las propuestas que adoptan referencias externas para la interpretación, situadas en el propio texto o más allá de él, se enfrentan a la cuestión de cómo restablecer el protagonismo del estudiante en el proceso de interpretación de su comprensión matemática. ¿Cómo recomponer el estatus cognitivo del alumno que comprende, cómo retornar de nuevo a su comprensión matemática reincorporándolo a él como protagonista de su propio proceso de interpretación tras el inevitable distanciamiento inicial? En el marco de la orientación semiótica, por ejemplo, donde el alejamiento resulta especialmente acentuado, se aprecia una clara renuncia al carácter mental de la comprensión. Por tanto, no hay intención de contemplar el retorno como tal, dado que la interpretación se circunscribe, exclusivamente, al análisis de la complejidad de las relaciones semióticas externas desplegadas durante la actividad matemática visible, sin más intervención complementaria del sujeto en el propio proceso interpretativo (OTTE, 2006).

Así pues, la cuestión del retorno pone de relieve el modo de hacer partícipe al estudiante de la interpretación de su propia comprensión matemática. Como opción, nos 
parece acertada la búsqueda de interpretaciones donde el distanciamiento con el estudiante sea transitorio, donde cobre mayor protagonismo la presencia y la participación del alumno en su proceso interpretativo y donde quede desestimado cualquier desequilibrio entre las partes intervinientes, en el sentido de alguna desigualdad manifiesta entre los protagonistas (estudiantes y agentes intérpretes de su comprensión) o pretensión de dominio/control sobre el alumno. Apostamos, en definitiva, por la configuración de interpretaciones de la comprensión en matemáticas más inclusivas con los estudiantes (BROWN, 2008).

A pesar de los avances alcanzados en la configuración de nuestro modelo interpretativo, en la actualidad aun no hemos proporcionado una respuesta completa, que podamos considerar satisfactoria, al problema del retorno a la comprensión matemática del estudiante a partir de la caracterización de los usos dados al conocimiento matemático durante su actividad en el aula. La conexión última en el ciclo interpretativo original entre los usos del conocimiento matemático y la propia comprensión matemática, todavía requiere mayor desarrollo y concreción (Figura 2).

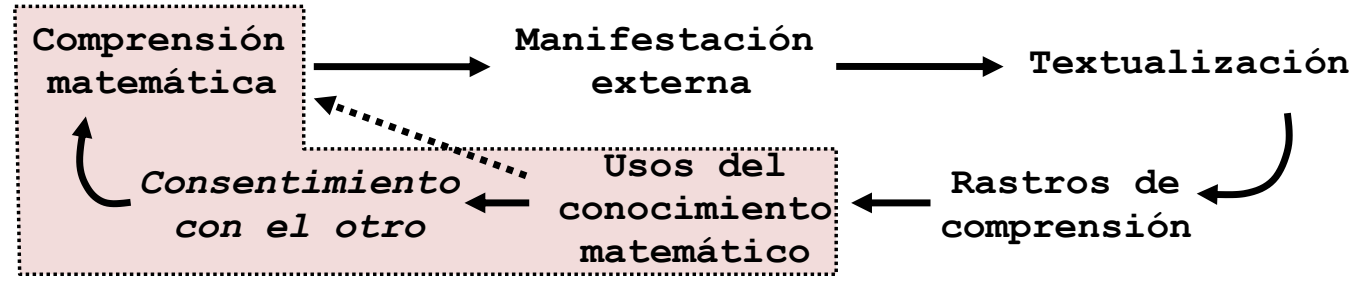

Figura 2 - Ciclo interpretativo de la comprensión en matemáticas

La cuestión es: ¿cómo recuperar el carácter cognitivo de la comprensión matemática si regresamos a ella con una interpretación sustentada en los usos externos del conocimiento matemático? Para responder a ello, proponemos una nueva extensión del ciclo, consistente en retornar a la comprensión matemática a través del consentimiento con el otro, un rastro visible complementario al uso que conecta y permite transitar desde el ámbito externo de los usos del conocimiento matemático a la esfera mental de la comprensión.

En estudios anteriores hemos realizado la interpretación de la comprensión matemática de los estudiantes a partir de caracterizaciones de los usos dados a los conocimientos matemáticos, tomando para ello como referencia análisis fenómeno-epistemológicos previos (GALLARDO; GONZÁLEZ， 2006; GALLARDO; GONZÁLEZ; QUISPE， 2008; GALLARDO; GONZÁLEZ; QUINTANILLA， 2013，2014a; ORTIZ; GONZÁLEZ; GALLARDO, 2012). Hasta la fecha, el objetivo ha sido identificar y delimitar, entre todo lo observado y registrado de la actividad matemática del estudiante, los rastros de su comprensión que pueden considerarse indicadores de algún uso dado al conocimiento 
matemático. En este punto, adoptamos una posición hermenéutica y sugerimos el intercambio discursivo entre participantes y el elemento común del consenso obtenido por ellos como añadido para respaldar la pertinencia de la interpretación. En términos estrictos, no nos bastamos solos para ver o decir algo sobre la comprensión matemática de los estudiantes.

Proponemos, ahora, continuar el proceso interpretativo con la búsqueda de una conformidad recíproca entre el propio estudiante y su agente intérprete (investigador/profesor/compañeros) acerca de las conclusiones sobre los usos del conocimiento matemático obtenidas en las fases precedentes del ciclo. En esta nueva fase, la comprensión interpretada se contrasta discutiendo con el propio alumno sobre los usos del conocimiento matemático evidenciado. Ahora, se trata de compartir y negociar las diferencias entre lo realizado por el estudiante y lo propuesto por el agente intérprete de su comprensión; de reconocer y asumir otras posibilidades, además de las propias sobre los usos del conocimiento matemático; de tomar conciencia mutua de la comprensión, propia y ajena, desplegada en el episodio. En esencia, se trata de alcanzar acuerdo, de lograr consenso, entre el alumno y sus interlocutores intérpretes acerca del trabajo matemático desempeñado. Es en este sentido que hablamos de alcanzar el consentimiento con el otro en el ejercicio de la interpretación de su comprensión matemática. Tanto la explicitación de la intención del alumno como la apropiación por parte del agente intérprete transcurre en la fase de consentimiento con el otro. En esta confluencia de la explicitación y la apropiación es donde se completa el círculo interpretativo de la comprensión en matemáticas que proponemos en nuestro modelo operativo. En la fase de consentimiento con el otro es donde también se plasma y percibe la dimensión o condición lingüística de toda experiencia matemática, una nueva puesta en valor de lo semiótico, esta vez a nivel discursivo.

La búsqueda de consentimiento está motivada por la necesidad de resolver ciertos desajustes o desequilibrios acontecidos durante la actividad matemática del estudiante que provocan algún tipo de incertidumbre en quien interpreta la comprensión en términos de usos dados a los conocimientos matemáticos. Es el caso, por ejemplo, de un registro escrito que evidencia el empleo de distintos conocimientos matemáticos que entran en conflicto o se interfieren entre sí durante la resolución de tal modo que encubren la comprensión desplegada por el alumno y dificultan su interpretación.

Proseguir con la fase de consentimiento también está justificado cuando no resulta suficiente la interpretación de la comprensión con base en los usos dados al conocimiento matemático, en el sentido de que no resuelve por sí misma estados de comprensión no deseables en el alumno. En nuestra opinión, una interpretación que evidencia una 
comprensión deficiente en el estudiante, en términos de usos inapropiados de los conocimientos matemáticos, al mismo tiempo debería asumir el compromiso de un esfuerzo suplementario, por parte de quien interpreta, destinado a revertir en lo posible tal situación. El consentimiento con el otro es nuestra propuesta para materializar esta intención solidaria e inclusiva que busca recobrar el protagonismo del estudiante, haciéndolo intervenir en el proceso de interpretación de su propia comprensión matemática.

La relación entre alumno y agente intérprete tiende a igualarse en esta fase, dado que ambos se necesitan para la interpretación. Por un lado, la obtención de consentimiento trae como consecuencia la mejora de la comprensión matemática del estudiante. En general, el agente intérprete ofrece una oportunidad para que el propio alumno desarrolle un aprendizaje con comprensión. Por otro lado, la interpretación de la comprensión matemática del estudiante exige su participación como mediador entre lo que él previamente ha realizado (el registro de la acción matemática acontecida) y el agente empeñado en concretar lo que comprende y cómo lo comprende. El alumno interviene, de forma directa, en la construcción del consentimiento durante su práctica matemática, por lo que se convierte en artífice directo y protagonista del proceso de interpretación de su propia comprensión. A través de la fase de consentimiento alcanzamos una mayor garantía sobre la comprensión matemática manifestada por el estudiante, al servir de complemento a la interpretación previa de los usos dados al conocimiento matemático.

En definitiva, el consentimiento con el otro nos ofrece una vía para retornar a la comprensión matemática. Por medio del protagonismo final adquirido por el estudiante, garantizamos, de nuevo, el carácter cognitivo de su comprensión, desplazado provisionalmente al comienzo del ciclo interpretativo, y completamos, de un modo más inclusivo, el ciclo interpretativo de la comprensión de la actividad matemática en el aula. La operatividad de nuestra propuesta pasa por evidenciar en la práctica la presencia del consentimiento con el otro en los procesos de interpretación. Perseguimos determinar de qué manera se construye y se logra este consentimiento durante la actividad matemática de los estudiantes. En la siguiente sección presentamos algunos ejemplos de ello.

\section{Presencia del consentimiento con el otro en la práctica: algunas evidencias empíricas}

La última fase en la interpretación de la comprensión, correspondiente al consentimiento con el otro, se manifiesta en la práctica. Lo evidenciamos aquí presentando dos ejemplos concretos tomados de la actividad matemática, escolar y cotidiana, de Inés y de 
Bing Bing, dos alumnas de primer curso de educación secundaria. Las directrices metodológicas de nuestro modelo operativo incluyen una estrategia combinada consistente en identificar y validar los rastros de comprensión matemática diseminados a lo largo del registro escrito, en primer lugar, y revelar en estos rastros los usos dados a los conocimientos matemáticos puestos en juego, en segundo lugar. La propia estructura fenómenoepistemológica del conocimiento se emplea como referencia objetiva para certificar tales usos (GALLARDO; GONZÁLEZ; QUINTANILLA, 2013, 2014a). En esencia, la fase del ciclo interpretativo previa al consentimiento con el otro tiene por objeto caracterizar los usos y relaciones presentes en los rastros de comprensión detectados en los fragmentos de actividad matemática.

En los dos casos concretos que nos ocupan, tomamos como punto de partida el registro escrito elaborado por cada alumna. En esta ocasión, intentan resolver una tarea convencional extraída de la unidad didáctica de su libro de texto dedicada al estudio de la divisibilidad de los números naturales. La tarea propuesta es la siguiente:

Los participantes en un desfile pueden colocarse, para desfilar, de 3 en 3, de 5 en 5 o de 25 en 25; pero no pueden hacerlo de 4 en 4 ni de 9 en 9. ¿Cuál es el número de participantes si sabemos que está entre 1000 y 1250? (COLERA; GAZTELU, 2011, p. 71).

En primer lugar, identificamos en cada registro escrito de lápiz y papel elaborado por las alumnas los diferentes rastros de comprensión que nos permiten caracterizar los usos dados a los conocimientos matemáticos durante la resolución. Una vez interpretada la comprensión desplegada por las alumnas en términos de usos dados al conocimiento matemático, regresamos a ellas, de nuevo, en una fase posterior, con la intención de compartir nuestra valoración sobre su desempeño en la tarea y alcanzar algún acuerdo acerca de su comprensión matemática. Con ello buscamos la conformidad recíproca en lo acontecido característica del consentimiento con el otro. Como la interpretación que proponemos se realiza sobre el registro escrito de toda manifestación observable, la fase de consentimiento, que transcurre en un contexto esencialmente verbal y discursivo, también es grabada en audio para su posterior textualización. A continuación, con base en los distintos registros escritos recopilados (productos en forma de respuestas de lápiz y papel y diálogos transcritos), mostramos las causas que provocaron la conveniencia de alcanzar este consentimiento y los detalles de cómo se fue forjando entre los protagonistas en cada uno de los casos analizados.

\subsection{El caso de Inés}


Del registro escrito identificamos distintos rastros de su uso del conocimiento matemático (Figura 3).

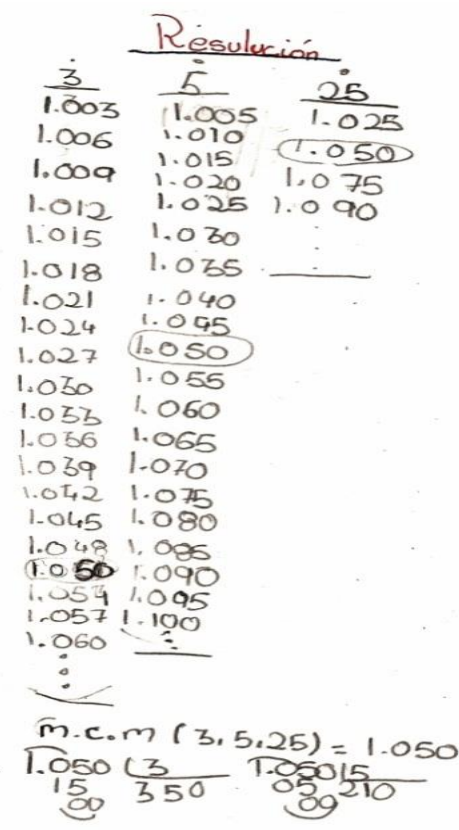

Figura 3 - Registro escrito de Inés

Por una parte, parece emplear la estrategia de resolución consistente en generar de inicio listados con cierta extensión de los múltiplos de 3, de 5 y de 25 mayores que mil, respectivamente, para después encontrar un número común en esos listados. En las tres listas aparece destacado el número 1050, rodeado con un círculo, lo que nos hace pensar que este número es su propuesta de solución para los participantes en el desfile. El carácter común del 1050 también queda expresado, aunque de forma incorrecta, en términos de mínimo común múltiplo de 3, de 5 y de 25. En todo caso, también incluye una comprobación de la divisibilidad de 1050 por 3 y por 5, utilizando para ello el algoritmo estándar escrito para la división de números naturales. Y este número común sería, para ella, el candidato a solución, aunque no comprueba la condición de no poder ser divisible por 4 ni por 9 .

Por otra parte, observamos dos errores en el listado de los múltiplos de 3. El primero consiste en comenzar con el número 1003 , a pesar de no ser un múltiplo de 3 , lo cual pone de manifiesto que no contempla el criterio de divisibilidad por 3 como elemento de prueba. El segundo lo encontramos más abajo con la propia presencia del 1050 en el listado. Y observamos, aquí, un desajuste en los criterios empleados para certificar la divisibilidad del 1050 por 3. Por un lado, al incluir 1050 en la lista, Inés reconoce que es un múltiplo de 3, aspecto que también es corroborado mediante la división por 3. Por otro lado, y precisamente 
por incluirlo, incumple la regla de contar de tres en tres a partir de 1003, un método que ha venido empleando para generar los múltiplos en los otros listados.

En definitiva, los usos dados a todos estos conocimientos matemáticos vinculados con la divisibilidad, así descritos, nos permiten interpretar hasta cierto punto la comprensión matemática de Inés. Encontramos muestras positivas, aunque no consolidadas, de su comprensión sobre lo que significa ser múltiplo de un número y también acerca de cómo generar múltiplos y cómo comprobar la divisibilidad de éstos. No percibimos, en cambio, una disposición clara para el empleo de criterios de divisibilidad como el del 3, lo cual nos impide pronunciarnos acerca de su comprensión sobre tales criterios. Los errores y desajustes evidenciados también generan incertidumbre en nuestra interpretación sobre otros aspectos concretos de su comprensión, como por ejemplo determinar qué criterio prevalece en ella para comprobar la divisibilidad de un número por otro (división exacta o criterios específicos). Estas últimas circunstancias son las que justifican, finalmente, la conveniencia de proseguir con el ciclo interpretativo en este caso.

\subsubsection{Discurso hacia el consentimiento}

Tomamos, ahora, la información anterior como referencia y nos introducimos con ella en la fase final del ciclo en busca del consentimiento con Inés. El siguiente fragmento (Cuadro 1) recoge la parte del discurso, entre investigador y alumna sobre los usos dados a los conocimientos matemáticos, donde se aprecia la evolución hacia el consentimiento alcanzado en esta ocasión:

\begin{tabular}{|c|c|c|}
\hline 1 & Investigador & $\begin{array}{l}\text { Vi que la respuesta estaba bien: 1050. Tú empezaste aquí a poner múltiplos de } \\
\text { 3, múltiplos de } 5 \text { y múltiplos de } 25 . \text { ¿Es así? }\end{array}$ \\
\hline 2 & Inés & Claro. \\
\hline 3 & Investigador & $\begin{array}{l}\text { Hemos quedado en que los múltiplos de } 3 \text { son 1003, 1006, 1009, 1012, ... ¿Estás } \\
\text { de acuerdo con eso? }\end{array}$ \\
\hline 4 & Inés & $\begin{array}{l}\text { No, porque es que si le sumas [en referencia a las cifras de 1009] te da } 10 . \\
\text { Entonces se divide por } 3 \text { y no da exacto [indicio de uso del criterio de } \\
\text { divisibilidad del número 3]. }\end{array}$ \\
\hline 5 & Investigador & La solución es correcta, que es 1050. ¿Dónde estaba el problema? \\
\hline 6 & Inés & $\begin{array}{l}\text { En que... Yo vi que } 5 \text { y } 25 \text { si daba correctamente el 1050. Y entonces, lo dividí } \\
\text { entre } 5 \text { y me daba exacta. Lo dividí entre } 25 \text {, me daba exacta. Y lo dividí entre } 3 \\
\text { y como me daba exacta, entonces yo he determinado que eso es así. }\end{array}$ \\
\hline 7 & Investigador & $\begin{array}{l}\text { Porque aquí yo veo que estás contando de tres en tres: } 3,6,9,12, \ldots \\
\text { ¿Eso es lo que te hizo poner el } 1050 \text { aquí? [El profesor se refiere a la división } \\
\text { exacta como criterio para comprobar que } 1050 \text { es múltiplo de } 3 \text { ]. }\end{array}$ \\
\hline 8 & Inés & Sí. \\
\hline 9 & Investigador & ¿Y por eso está como medio borrado? \\
\hline
\end{tabular}




\begin{tabular}{|lll|}
\hline 10 & Inés & Sí \\
11 & Investigador & ¿Antes qué debería haber? \\
12 & Inés & 51 \\
13 & Investigador & $\begin{array}{l}\text { Antes había un 1051 porque ibas contando de tres en tres. Entonces hiciste ahí } \\
\text { un arreglo para que diese y esa fue tu solución. }\end{array}$ \\
14 & Inés & [Risas con asentimiento]
\end{tabular}

Cuadro 1 - Fragmentos del diálogo entre investigador e Inés

Coincidimos con Inés respecto a su intención de determinar listados y utilizar el conteo equitativo como método para generar múltiplos de 3 , de 5 y de 25 , respectivamente (líneas 1 y 2). En el caso de la lista correspondiente al número 3, termina por reconocer que los sucesivos números obtenidos a partir de 1003, contando de tres en tres, no son en realidad múltiplos de 3 por no cumplir el criterio de divisibilidad por 3 (la suma de las cifras del número debe ser múltiplo de 3). Esto lo interpretamos como una muestra positiva, no evidenciada con anterioridad, de la comprensión de Inés acerca de los criterios de divisibilidad (3-4).

Por otra parte, como ya interpretábamos, Inés llega a considerar el 1050 múltiplo de 3 como consecuencia de la comprobación de una división exacta. Sin embargo, más allá de lo apreciado en su registro escrito, ahora nos revela que la candidatura de 1050 para ser incluido también en el listado de múltiplos de 3 fue previa a su comprobación efectiva y estuvo motivada al observar que ya era múltiplo de 5 y de 25. Esta última consideración completa nuestra interpretación sobre su elección de 1050 como solución del problema (5-6). Para concluir la interpretación, tan solo resta acordar con Inés la razón de la inclusión de 1050 en el primer listado. A partir de 1003 va generando sucesivos múltiplos de 3, contando de tres en tres, pero no alcanza el 1050 como consecuencia del error inicial reconocido por ella, sino que obtiene el 1048 y de aquí pasa al 1051. Como ella ya sabe que 1050 es múltiplo común de los tres listados, para hacer efectiva su estrategia y lograr que todo cuadre se ve obligada a ajustar el listado de múltiplos de 3, sustituyendo el número 1051 por el deseado 1050 (7-14). En definitiva, impone al listado su consideración del 1050 como múltiplo de 3 argumentada mediante una vía externa al listado de múltiplos de 3 y con métodos directos (división) de comprobación de la divisibilidad en lugar del uso de criterios específicos para ello.

\subsubsection{Sobre la comprensión matemática de Inés}

Una vez concluida la fase de consentimiento, ¿qué podemos afirmar acerca de la comprensión de Inés sobre la divisibilidad de números naturales? Obtenemos indicios 
razonables de una comprensión de la divisibilidad que podríamos valorar como incipiente y en clara evolución. Dicha comprensión está sustentada, sobre todo, en los conceptos de múltiplo y divisor, a través de sus definiciones estándar, así como en el uso de los correspondientes métodos directos de comprobación de la divisibilidad que se desprenden de tales definiciones. Asimismo, la comprensión de estos conocimientos, que son propios en el inicio del estudio de la divisibilidad, se extiende además sobre algunos de los criterios específicos de divisibilidad más usuales, como los del 3 o el 5. No obstante, a nuestro juicio la comprensión de estos criterios se muestra en Inés de un modo más débil o inestable en comparación con la de los primeros conocimientos.

\subsection{El caso de Bing Bing}

El protocolo que proporciona Bing Bing (Figura 4) contribuye a mejorar nuestra interpretación basada en los usos dados a los conocimientos matemáticos.

\begin{tabular}{|c|c|}
\hline 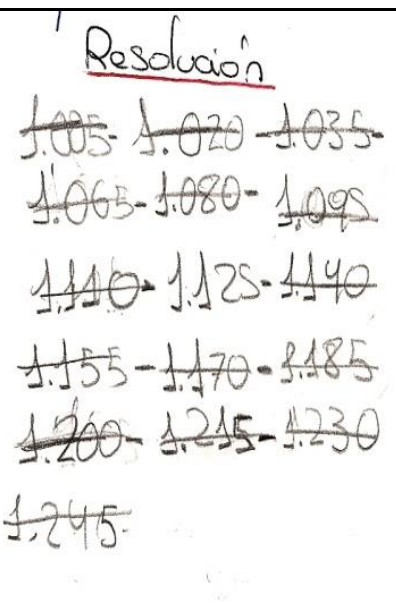 & $\begin{array}{l}\text { Primero escribo los números que acaben en } 0 \text { o en } 5 \text {, } \\
\text { porque dice que es divisible entre } 5 \text { y también los que } \\
\text { sean divisibles entre } 3 \text {. } \\
\text { Ahora pruebo si son divisibles en } 25 \text {, porque dice que } \\
\text { pueden colocarse de } 25 \text { en 25, que son los que terminan } \\
\text { en } 25 \text { o } 00 \text {. } \\
\text { En el enunciado dice que no pueden agruparse de } 4 \text { en } 4 \\
\text { ni de } 9 \text { en 9, por eso } 1200 \text { no puede ser, porque sus dos } \\
\text { primeras cifras son divisibles entre } 4 \text {, es decir, que es un } \\
\text { número divisible entre } 4 \text {. } \\
\text { La solución es } 1125 \text {. Porque cumple todas las } \\
\text { condiciones. }\end{array}$ \\
\hline
\end{tabular}

Figura 4 - Registro escrito de Bing Bing con transcripción del protocolo explicativo

En concreto, sustenta la elaboración de su respuesta en el uso permanente de criterios específicos de divisibilidad. Comienza elaborando la lista de los números entre 1000 y 1250 que son, simultáneamente, múltiplos de 5 y de 3, utilizando para ello los criterios de divisibilidad de 5 (todos los números propuestos terminan en 0 o en 5 ) y de 3 (la suma de las cifras de cada número candidato es múltiplo de 3). El listado, sin embargo, está incompleto porque falta, única y precisamente, el número 1050. Podría contemplarse como un descuido cometido por Bing Bing durante la resolución de la tarea.

A continuación, sobre el conjunto resultante de números candidatos, aplica un nuevo criterio de divisibilidad, el correspondiente al número 25. Procede con el descarte tachando los números que no cumplen $s u$ regla para ser divisible por 25: aquellos números que 
terminan en 25 o 00. En esta ocasión, se trata de una variante personal incorrecta del criterio: un número es divisible por 25 cuando sus dos últimas cifras son cero o constituyen un múltiplo de 25. Tras esta comprobación, sus candidatos se reducen a dos: el 1125 y el 1200 . Como no pueden ser divisibles por 4 ni por 9, suprime el número 1200 como consecuencia de utilizar, en este caso, el criterio de divisibilidad específico para el número 4: un número es divisible por 4 cuando sus dos últimos dígitos son ceros o forman un múltiplo de 4 . Finalmente, la propuesta de 1125 como solución final para el número de participantes en el desfile también se ve afectada por la no comprobación de su divisibilidad por 9.

En definitiva, el registro escrito interpretado en este episodio nos aporta rastros claros de la comprensión de Bing Bing sobre la divisibilidad de los números naturales. Sobre todo, la disponibilidad general al empleo de criterios de divisibilidad y los usos pertinentes dados a los criterios del 3, del 5 y del 4, son indicadores positivos de la solidez de su comprensión sobre estas reglas específicas. Por otra parte, también se desprende de su resolución algunos usos alterados que condicionan la idoneidad de su respuesta final. Nos referimos a (a) la ausencia del número 1050 en el listado inicial de múltiplos de 5 y de 3; (b) la adaptación del criterio de divisibilidad de 25 , que no contempla los números cuyas últimas dos cifras son múltiplos de 25 (como 1050, precisamente); y (c) la omisión de la comprobación de la divisibilidad de 1125 por 9 . Estos son los principales desajustes que motivan, en esta ocasión, la búsqueda de consentimiento con Bing Bing.

\subsubsection{Discurso hacia el consentimiento}

Además de contrastar nuestra interpretación, realizada con base en los distintos usos ya mencionados, nuestros esfuerzos en esta fase del ciclo interpretativo van dirigidos, fundamentalmente, a lograr que la alumna se percate de lo realizado, tome conciencia de la necesidad de modificar algunos aspectos de su procedimiento, proponga alternativas compatibles con las expectativas de sus interlocutores $y$, finalmente, alcance un acuerdo con ellos acerca de la actividad matemática desarrollada. Tras recorrer conjuntamente con Bing Bing los pasos de su resolución, siguiendo el protocolo proporcionado, y compartir con ella nuestra interpretación de lo realizado, la búsqueda de consentimiento prosigue del siguiente modo (Cuadro 2). 


\begin{tabular}{|c|c|c|}
\hline 1 & Investigador & $\begin{array}{l}\text { Pero creo que te olvidaste probar si el } 1125 \text { es divisible por } 9 \text { o no. Y } 1125 \text { me } \\
\text { parece que es divisible por } 9 \text {. }\end{array}$ \\
\hline 2 & Bing Bing & ¡Entonces lo tengo mal! \\
\hline 3 & Investigador & ¿Y qué hacemos? \\
\hline 4 & Bing Bing & ¡Pues dejarlo así! \\
\hline
\end{tabular}

Cuadro 2 - Fragmentos del diálogo entre investigador y Bing Bing

La pretensión de Bing Bing de buscar consenso mediante la simple aceptación pasiva de las sugerencias de su interlocutor denota conformidad con lo realizado y persigue el abandonando de la tarea (2-4). Se trata de una propuesta de acuerdo matemáticamente insatisfactoria para ambos. Nuestro modelo interpretativo no admite solventar de este modo la fase de consentimiento, dado que la disposición actual de la alumna no contribuye a modificar su propia comprensión matemática, ni tampoco satisface nuestras expectativas de retorno a ella. Con el fin de reconducir la posición inicial de Bing Bing y avanzar en la interpretación, en este punto abrimos el discurso a dos de sus compañeras, una de ellas Inés, que alcanzaron por otras vías la solución correcta al problema (Cuadro 3).

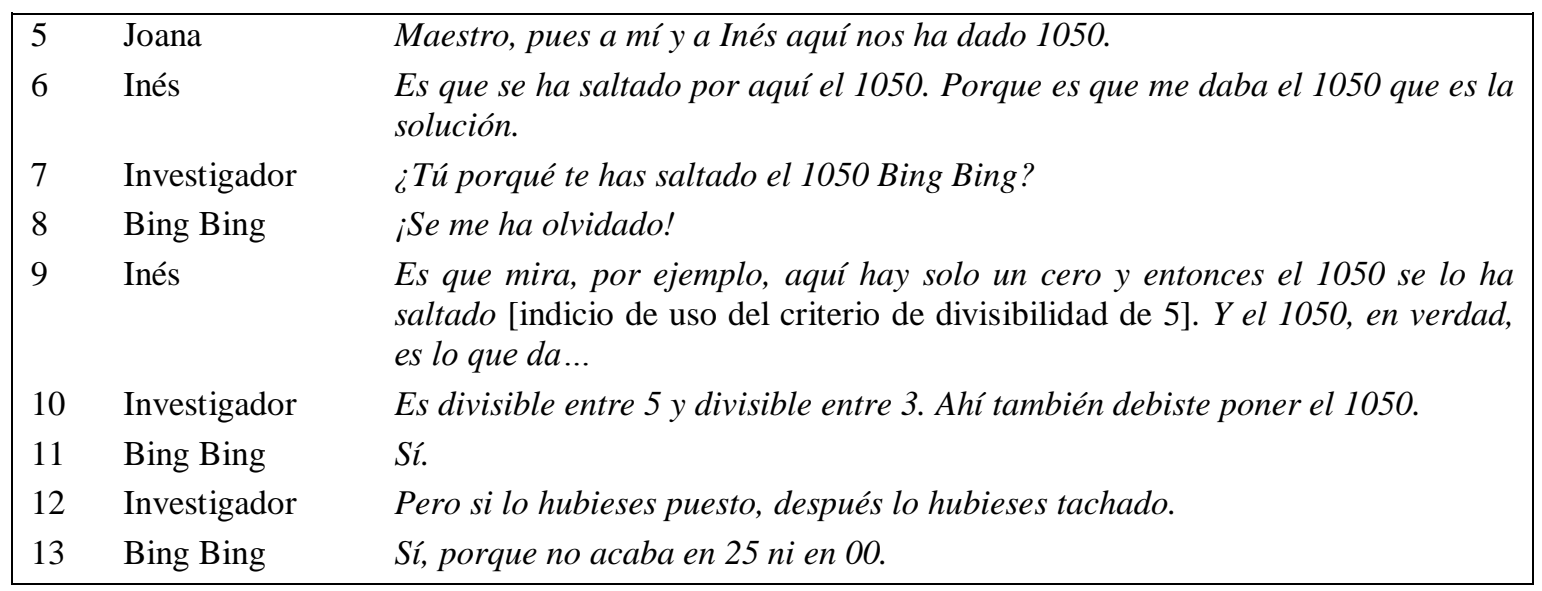

Cuadro 3 - Fragmentos del diálogo entre el investigador y las alumnas (líneas 5-13)

La intervención de sus compañeras pone en evidencia el olvido de incluir el número 1050 en el listado inicial de múltiplos de 5 y de 3 (5-8). La explicación de Inés, que aporta un nuevo indicio de su propia comprensión de la divisibilidad a través del uso del criterio correspondiente al 5, contribuye sobre todo a que Bing Bing reconozca, de forma justificada (no sólo por simple aceptación), que en principio 1050 también es candidato (9-11). Esto es, en este momento consideramos que la alumna ya es consciente de que 1050 es múltiplo de 5 y de 3, al cumplir los requisitos impuestos por los correspondientes criterios de divisibilidad. Y lo interpretamos como unos primeros indicios positivos de consentimiento que Bing Bing sustenta en su propia comprensión matemática. No obstante, enseguida descarta la posibilidad de 1050 como solución por volver a utilizar su versión personal del criterio de divisibilidad de 25: 1050 no es divisible por 25 porque no acaba en 25 ni en 00 (12-13). Ahora, con el número 
1050 presente al menos en la discusión, la atención se dirige hacia la reflexión sobre la idoneidad del propio criterio de divisibilidad de 25. Aquí, la participación de las compañeras de Bing Bing vuelve a ser decisiva (Cuadro 4).

\begin{tabular}{|lll|}
\hline 14 & Joana & Pero, ¿y qué tiene que ver que acabe en 25 o en 00? \\
15 & Bing Bing & Es que yo creo que se me han olvidado los criterios de divisibilidad. \\
16 & Investigador & ¿Cuál? ¿El del 25? \\
17 & Bing Bing & Sí \\
18 & Inés & Es que el del 25 no lo dimos. \\
19 & Investigador & ¿Qué problema hay? ¿Dónde está el problema? \\
20 & Bing Bing & Me he equivocado con el criterio de divisibilidad del 25. \\
21 & Investigador & ¿Estáis de acuerdo con eso? \\
22 & Bing Bing, Inés & Sí. [Respuesta conjunta] \\
& y Joana & El haberle hecho demasiado caso al criterio del 25 es lo que ha hecho que tu \\
23 & Investigador & argumento se haya ido por otro lado. En el momento en que lo quitamos, todo \\
& & cuadra. Bueno, siempre que hayas puesto... \\
24 & Bing Bing & El lo50.
\end{tabular}

Cuadro 4 - Fragmentos del diálogo entre el investigador y las alumnas (líneas 14-24)

La pregunta de Joana provoca en Bing Bing falta de convicción en la idoneidad matemática de su criterio de divisibilidad de 25 (14-18). De hecho, parece anteponer ya la opción de 1050 como candidato a solución frente a su versión de no ser divisible por 25, logrando, de este modo, un nuevo consentimiento con su profesor y compañeras (19-24). En resumen, en este episodio el discurso sobre cuatro hechos matemáticos concretos contribuyó al logro del consentimiento con el otro: (a) la divisibilidad por 9 del número 1125, que lo descarta como solución del problema; (b) la necesaria inclusión del número 1050 en el listado inicial; (c) su condición de ser múltiplo de 5 y de 3; y (d) la versión alterada del criterio de divisibilidad de 25. Lo relevante, aquí, es que todos estos hechos fueron reconocidos y admitidos por Bing Bing con base en una fundamentación que dio muestras de estar sustentada en su propia comprensión de la divisibilidad de los números naturales.

\subsubsection{Sobre la comprensión matemática de Bing Bing}

Bing Bing manifiesta una clara predisposición para el empleo de los criterios de divisibilidad, dado que sustenta la resolución de la tarea mayoritariamente en ellos. La especificidad de estos criterios, unida a una fundamentación matemática compleja en la mayoría de los casos, los hace ser conocimientos matemáticos más sofisticados en comparación con los procedimientos directos que se derivan del empleo de las definiciones vinculadas con la divisibilidad. La inclinación natural de la alumna al uso de tales criterios, 
aun siendo de un modo meramente técnico, la interpretamos como una muestra positiva de comprensión sólida acerca de la divisibilidad de los números naturales. En esta faceta concreta de la divisibilidad, podemos afirmar que Bing Bing posee una comprensión más evolucionada que la evidenciada, por ejemplo, por su compañera Inés. La fase de consentimiento también nos ha permitido observar cómo esta alumna ha sido capaz de reconducir la resolución de la tarea, modificando algunos usos propios alterados, con una solvencia destacada que atribuimos a la consistencia de su comprensión matemática.

Es decir, consideramos que Bing Bing alcanza la solución del problema propuesto como consecuencia exclusiva de la comprensión matemática que posee sobre la divisibilidad de los números naturales. Una comprensión que no tiene dificultad en ajustar a los requerimientos del problema cuando la exterioriza en su búsqueda de consentimiento y que, finalmente, le resulta suficiente para la ocasión. También hemos percibido cómo la búsqueda de consentimiento mediante argumentaciones sustentadas en la comprensión matemática motivó un cambio favorable en su predisposición a resolver la tarea. Durante el transcurso de la discusión, la alumna pasó de una aceptación pasiva (2-4) y una justificación superficial (78) con connotaciones afectivas negativas a un reconocimiento y modificación positiva de su desempeño en sintonía con sus compañeras (20-22). De este modo, la comprensión de Bing Bing se mostró condicionante de la faceta afectiva de su propio desempeño matemático, en el sentido de que contribuyó a su desarrollo a través de la búsqueda de consentimiento.

\section{Acceso a la faceta socioafectiva de la comprensión en matemáticas a través del consentimiento}

Toda actividad matemática está propiciada por, y es consecuencia de, acciones intelectuales que demandan unas exigencias cognitivas necesariamente vinculadas a la esfera mental individual de sus protagonistas. En nuestro modelo interpretativo tomamos como referencia este supuesto y concebimos la comprensión en matemáticas como un fenómeno mental de carácter cognitivo, como una actividad intelectual cognitiva específica, experimentada como tal exclusivamente por quien la desarrolla. Esta actividad mental capacita o hace competente al individuo para elaborar respuestas, adaptadas y contextualizadas, que involucran la utilización del conocimiento matemático en alguna de las categorías y formas posibles de su dimensión fenómeno-epistemológica (GALLARDO; GONZÁLEZ; QUINTANILLA, 2013, 2014a). 
Al mismo tiempo, desde una perspectiva más social, también reconocemos que la interacción en el aula y la toma de conciencia de la actividad matemática configuran escenarios de interpretación de la comprensión matemática mediados por el contexto social y cultural (BROWN, 2008). Las interacciones de los participantes en tales escenarios proporcionan rastros de comprensión colectiva, donde intervienen distintas formas de ver y comprender las matemáticas relacionadas y orientadas hacia el acuerdo social (MARTIN; TOWERS, 2003; RESÉNDIZ, 2010).

La consideración conjunta de tales posicionamientos respecto a la comprensión matemática, individual y social, nos plantea la cuestión de su relación en el momento de la interpretación: ¿Cómo conciliar en el ejercicio de la interpretación el carácter personal que otorgamos a la comprensión matemática con su desarrollo compartido en entornos socioculturales? Con intención integradora, la fase de consentimiento con el otro nos ofrece la posibilidad de responder con una propuesta operativa.

Las componentes social e individual de la comprensión en matemáticas se muestran estrechamente vinculadas en la fase de consentimiento. En principio, el camino hacia el consentimiento lo consideramos de naturaleza esencialmente discursiva, tal como lo ejemplifican los casos prácticos interpretados. Es una fase que permite apreciar la dimensión sociocultural de la actividad matemática por cuanto en ella afloran las interacciones personales y el conocimiento matemático emerge y evoluciona como consecuencia de un discurso contextualizado y colaborativo entre el alumno y los intérpretes de su comprensión (BARBOZA; DO REGO; BARBOSA, 2013). No obstante, las interacciones y réplicas discursivas entre los interlocutores no garantizan por sí mismas el logro del consentimiento en esta fase. El consentimiento está mediatizado, sobre todo, por la comprensión matemática individual del alumno, en el sentido de que se ejerce y se alcanza siempre a través de y con la aprobación de ésta. Esto es, el estudiante es el principal legitimado para concluir con la fase de consentimiento y asignar un grado de satisfacción a lo realizado, basándose para ello en su propia comprensión de los conocimientos desplegados durante la actividad matemática.

Los ejemplos descritos nos han ofrecido la oportunidad de apreciar cómo la posibilidad de lograr algún tipo de consentimiento está supeditada a las particularidades de la comprensión que, en este caso particular, poseen las dos alumnas analizadas sobre conocimientos matemáticos vinculados con la divisibilidad de los números naturales. Por tanto, las interacciones discursivas acontecidas en el transcurso de esta fase también dependen de la comprensión matemática de las alumnas. En definitiva, en la fase de consentimiento, aun siendo esencialmente discursiva y social, siempre habrá una elaboración intelectual, 
individual e interna por parte del alumno, condicionante de los aspectos discursivos y sociales porque es el estudiante, en última instancia, quien pone en juego su propia compresión de los conocimientos matemáticos para resolver la situación problemática.

La influencia de la comprensión en esta fase alcanza, incluso, al ámbito afectivo de las matemáticas. En Educación Matemática se admite la existencia de una profunda interrelación entre afectividad y cognición. Mayoritariamente, son reconocidas las influencias afectivas sobre el procesamiento cognitivo, en el sentido de que los fenómenos emocionales y motivacionales condicionan el pensamiento y el aprendizaje matemático (LEDER, 2006; CARNEIRO; FRADE; DA ROCHA, 2010). En este trabajo hemos constatado una relación recíproca y complementaria a ésta: en la búsqueda de consentimiento con el otro, el afecto evoluciona condicionado por la comprensión matemática del estudiante.

Los ejemplos descritos nos han aportado indicios favorables de cómo la fase de consentimiento contribuye en la práctica a la interpretación y desarrollo de la faceta afectiva de la comprensión matemática. El asentimiento complaciente de Inés, al final de su diálogo, o la evolución positiva experimentada por Bing Bing respecto a su compromiso por encontrar una solución acorde con la de sus interlocutores, acontecieron esencialmente como consecuencia directa de su comprensión matemática personal. En este sentido, la comprensión matemática vuelve a ofrecernos a través del consentimiento una vía operativa para transitar esta vez hacia la interpretación del afecto en matemáticas.

\section{Consideraciones finales}

En el ciclo interpretativo de la comprensión en matemáticas hemos incorporado la fase de consentimiento con el otro con el propósito principal de dotar de sentido al tránsito entre los usos del conocimiento matemático y la propia comprensión matemática del alumno. Las interacciones discursivas que evidencian el consentimiento contribuyen a la interpretación con nuevos rastros de comprensión complementarios a los usos del conocimiento matemático. De este modo, situamos la referencia para la interpretación de la comprensión en matemáticas en el propio conocimiento matemático, a través de los usos dados y de los consentimientos adquiridos durante la actividad matemática.

En los ejemplos analizados hemos constatado que la comprensión matemática condiciona y determina, en última instancia, el desarrollo del consentimiento. Esta circunstancia consolida aun más el protagonismo del estudiante en el proceso de interpretación de su propia comprensión. Además de ello, la comprensión matemática también 
influye en la faceta social y afectiva de su interpretación, en el sentido de que los discursos y los afectos evolucionan condicionados por la comprensión propia del individuo. Ello nos hace reconocer una mediación de la comprensión sobre los aspectos sociales, discursivos y afectivos de la actividad matemática en el aula. Como consecuencia para la práctica docente, consideramos que nuestra propuesta ofrece una vía de acceso operativa a la interpretación y desarrollo de la faceta socioafectiva de la actividad matemática a través de la búsqueda del consentimiento con el otro y siempre con base en la comprensión matemática del estudiante.

Hasta ahora, hemos propuesto los análisis epistemológicos y fenomenológicos de los conocimientos matemáticos como referencia objetiva con la que dar cuenta en términos organizativos y clasificatorios de los usos observados en la actividad matemática del estudiante (GALLARDO; GONZÁLEZ; 2006; GALLARDO; GONZÁLEZ; QUISPE, 2008; GALLARDO; GONZÁLEZ; QUINTANILLA, 2013). La operatividad de nuestra propuesta depende, ahora, de la búsqueda de nuevas estructuras organizativas para el consentimiento con el otro sustentadas en el conocimiento matemático, similares a las elaboradas en la dimensión fenómeno-epistemológica de nuestro modelo interpretativo.

Nos queda pendiente la labor de establecer criterios y tipologías para el consentimiento que permitan alcanzar sistematicidad y uniformidad en las interpretaciones de la comprensión matemática en la fase final de nuestro ciclo interpretativo. Es precisamente aquí donde situamos el punto de partida para el desarrollo de una dimensión socioafectiva aun por explorar en nuestro modelo operativo para la interpretación de la comprensión en matemáticas, complementaria a las dimensiones fenómeno-epistemológica y hermenéutica ya existentes.

\section{Referencias}

BARBOZA, P. L.; DO REGO, R. M.; BARBOSA, J. C. Discursos do professor de matemática e suas implicações na compreensão dos alunos. Bolema, Rio Claro, v. 27, n. 45, p. 55 - 74, abr. 2013.

BARMBY, P.; HARRIES, T.; HIGGINS, S.; SUGGATE, J. How can we assess mathematical understanding? In: CONFERENCE OF THE INTERNATIONAL GROUP FOR THE PSYCHOLOGY OF MATHEMATICS EDUCATION, $31^{\text {st }}$, 2007, Seoul . Proceedings... Seoul: PME, 2007, v. 2, p. 41-48.

BROWN, T. Towards a hermeneutical understanding of mathematics and mathematical learning. In: ERNEST, P. (Ed.). Constructing mathematical knowledge: Epistemology mathematical education London: RoutledgeFalmer, 1996. p. 141-150.

BROWN, T. Mathematics education and language. Interpreting hermeneutics and poststructuralism. Dordrecht: Kluwer Academic Publishers, 2001. 
BROWN, T. Making mathematics inclusive: interpreting the meaning of classroom activity. Philosophy of Mathematics Education Journal, Exeter, n. 23, oct. 2008. Disponible at: <http://people.exeter.ac.uk/PErnest/pome23/index.htm>. Accessed on: 16 jun. 2014.

CARNEIRO, M.; FRADE, C.; DA ROCHA, J. T. Influência de aspectos afetivos na relação entre professor e alunos em sala de aula de matemática. Bolema, Rio Claro, v. 23, n. 36, p. 683-713, 2010.

COLERA, J.; GAZTELU, I. Matemáticas 1. Educación Secundaria. Madrid: Anaya, 2011.

DANCY, J. Introducción a la epistemología contemporánea. Madrid: Tecnos, 1993.

DUVAL, R. A cognitive analysis of problems of comprehension in a learning of mathematics. Educational Studies in Mathematics, Berlin, v. 61, n. 1/2, p. 103-131, 2006.

ELL, F. Can moderate hermeneutics help us to understand learning and teaching in the mathematics classroom? In: CONFERENCE OF THE INTERNATIONAL GROUP FOR THE PSYCHOLOGY OF MATHEMATICS EDUCATION, $30^{\text {th }}$, 2006, Prague, Czech Republic. Proceedings... Prague: PME, 2006, v. 3, p. 33-40.

FONT, V.; GODINO, J. D.; D'AMORE, B. An onto-semiotic approach to representations in Mathematics Education. For the Learning of Mathematics, Edmonton, v. 27, n. 2, p. 2-9, jul. 2007.

GALLARDO, J.; GONZÁLEZ, J. L. Assessing understanding in mathematics: steps towards an operative model. For the Learning of Mathematics, Edmonton, v. 26, n. 2, p. 10-15, jul. 2006.

GALLARDO, J.; GONZÁLEZ, J. L.; QUINTANILLA, V. A. Tareas, textos y usos del conocimiento matemático: aportes a la interpretación de la comprensión desde el cálculo aritmético elemental. Educación Matemática, México, v. 25, n. 2, p. 61-88, ago. 2013.

GALLARDO, J.; GONZÁLEZ, J. L.; QUINTANILLA, V. A. Sobre la valoración de la competencia matemática: claves para transitar hacia un enfoque interpretativo. Enseñanza de las Ciencias, Barcelona, v. 32, n. 3, p. 263-280, nov. 2014a.

GALLARDO, J.; GONZÁLEZ, J. L.; QUINTANILLA, V. A. Revisiting understanding in mathematics. Quadrante, Lisboa, v. 23, n. 1, p. 63-78, jun. 2014b.

GALLARDO, J.; GONZÁLEZ, J. L.; QUISPE, W. Interpretando la comprensión matemática en escenarios básicos de valoración. Un estudio sobre las interferencias en el uso de los significados de la fracción. Revista Latinoamericana de Investigación en Matemática Educativa, México, v. 11, n. 3, p. 355-382, nov. 2008.

GODINO, J. D. Un enfoque ontológico y semiótico de la cognición matemática. Recherches en Didactique des Mathématiques, Grenoble, v. 22, n. 2/3, p. 237-284, 2002.

GODINO, J. D.; BATANERO, C.; FONT, V. The onto-semiotic approach to research in Mathematics Education. The International Journal on Mathematics Education ZDM, Karlsruhe, v. 39, n. 1/2, p. 127-135, mar. 2007.

GOLDIN, G. Representation in mathematical learning and problem solving. In: ENGLISH, L. D. (Ed.). Handbook of international research in Mathematics Education. Mahwah: Lawrence Erlbaum Associates, 2002. p. 197-218.

HABERMAS, J. Teoría de la acción comunicativa I. Madrid: Taurus, 1999. 
LEDER, G. C. Affect and mathematics learning. In: MAASZ, J.; SCHOEGLMANN, W. (Ed.). New mathematics education research and practice. Rotterdam: Sense Publishers, 2006. p. 203-208.

MARTIN, L. C.; TOWERS, J. Collective mathematical understanding as an improvisational process. In: CONFERENCE OF THE INTERNATIONAL GROUP FOR THE PSYCHOLOGY OF MATHEMATICS EDUCATION, $27^{\text {th }}$, 2003, Honolulu, HI. Proceedings... Honolulu: PME, 2003, v. 3, p. 245-252.

MORGAN, C.; WATSON, A. The interpretive nature of teacher's assessment of students' mathematics: issues for equity. Journal for Research in Mathematics Education, Reston, v. 33, n. 2, p. 78-111, 2002.

ORTIZ, A. L.; GONZÁLEZ, J. L.; GALLARDO, J. Comprensión del sistema de numeración decimal en estudiantes del Grado de Primaria. In: MARÍN, M.; CLIMENT, N. (Coord.). Investigación en Educación Matemática. Comunicaciones de los grupos de investigación. XV Simposio de la SEIEM. Ciudad Real: SEIEM, 2012. p. 309-378.

OTTE, M. Proof and explanation from a semiotic point of view. Revista Latinoamericana de Investigación en Matemática Educativa, México, v. 9, n. 4, p. 23-43, 2006.

POPPER, K. El mito del marco común. Barcelona: Paidós, 2005.

RESÉNDIZ, E. El discurso en la clase de matemáticas y los acuerdos sociales. La noción de variación. Revista Latinoamericana de Investigación en Matemática Educativa, México, v. 13, n. 4-I, p. 99$112,2010$.

RICEEUR, P. Del texto a la acción. México: Fondo de Cultura Económica, 2002.

SÁENZ-LUDLOW, A.; ZELLWEGER, S. The teaching-learning of mathematics as a double process of intra- and inter-interpretation: A peircean perspective. In: INTERNATIONAL CONGRESS ON MATHEMATICAL EDUCATION ICME, $12^{\text {th }}, 2012$, Seoul, Korea. Proceedings... Seoul: ICME, 2012. p. 3117-3126.

TAHTA, D. On interpretation. In: ERNEST, P. (Ed.). Constructing mathematical knowledge: Epistemology mathematical education. London: RoutledgeFalmer, 1996. p. 125-133.

Submetido em Outubro de 2014. Aprovado em Abril de 2015. 